\title{
Stratégies biologiques à l'heure de l'Assistance Médicale à la Procréation chez le blessé médullaire
}

\author{
Manuel DEMAILLY1, Henri COPIN2, Philippe MERVIEL2, \\ Jacques PETIT1, Isabelle HENRY2
}

1 Service d'Urologie et Transplantation, et

2 Service de Biologie de la Reproduction et de Cytogénétique, $\mathrm{CHU}$, Amiens

\begin{abstract}
RESUME
L'incidence du nombre de blessés médullaires est en constante augmentation et parmi eux $41 \%$ ont moins de 30 ans. Les possibilités de procréation sont des préoccupations essentielles chez ces jeunes patients.

L'impact délétère du traumatisme médullaire sur la fonction éjaculatoire et sur les principaux paramètres spermatiques sont bien documentés : le volume, la numération et la mobilité sont perturbés.

Des congélations de sperme dites « préventives " sont à envisager rapidement après le traumatisme.

La conduite à tenir chez ces patients lésés médullaires qui désirent un enfant va dépendre de leurs capacités éjaculatoires et du nombre de spermatozoïdes mobiles obtenus.

Si une éjaculation est possible naturellement ou à l'aide de techniques simples (vibromassage) et que le sperme présente des caractéristiques normales une conception pourra être tentée à domicile.

Si les caractéristiques spermatiques sont mauvaises, des techniques d'AMP sont envisageables. En fonction du nombre de spermatozoïdes mobiles recueillis le couple bénéficiera d'une insémination intra-utérine, d'une fécondation in vitro ou d'une injection intracytoplasmique.
\end{abstract}

Mots-clés : blessé médullaire, procréation médicalement assistée, éjaculation, autoconservation

\section{INTRODUCTION}

L'incidence des traumatismes médullaires est en nette augmentation ces dernières années. Environ 1000 nouveaux cas sont dénombrés par an en France métropolitaine et la majorité d'entre eux concerne l'adolescent et l'adulte jeune ; les hommes étant plus touchés que les femmes [11].

Bien que les accidents de la voie publique demeurent une cause relativement fréquente, ce sont les accidents liés à une activité sportive (accidents de ski, de plongeoir,...) qui sont les plus fréquentes.

Etant donné le jeune âge de ces patients, la question de la procréation est une préoccupation clairement et rapidement exprimée au décours des suites traumatiques.

Afin de réaliser son désir d'enfant, le blessé médullaire de sexe masculin rencontre deux types de problèmes organiques: d'une part la difficulté à obtenir une éjaculation efficace et d'autre part une altération de la qualité de son sperme.

L'impact délétère du traumatisme médullaire sur la fonction éjaculatoire, la spermatogenèse et la qualité spermatique est actuellement bien connu et documenté.

\section{HISTORIQUE}

L'induction artificielle d'une éjaculation chez un blessé médullaire a eu lieu pour la première fois en 1947, grâce à l'injection intra-thécale de Néostigmine.

Les deux principales méthodes mécaniques de recueil de sperme employées de nos jours sont l'électroéjaculation (EEJ) utilisée depuis 1948 [13], et le vibromassage depuis 1965 [23].

\section{Correspondance :}

Dr Manuel DEMAILLY - Service d'Urologie, Hôpital Sud, CHU Amiens, 80054 Amiens cedex 1 -

email : demaillym@yahoo.fr 
La première grossesse par EEJ avec insémination intra-utérine a été rapportée en 1975 [24], et la première faisant suite à une EEJ avec fécondation in vitro date de 1988 [1].

\section{QUALITE SPERMATIQUE CHEZ LE BLESSE MEDULLAIRE}

Différentes atteintes de la lignée germinale testiculaire chez le blessé médullaire sont possibles et ont été décrites, expérimentalement et cliniquement.

\section{Lésions histologiques}

Peu d'études histologiques ont montré l'effet délétère de la lésion médullaire sur la spermatogenèse. Pour des raisons évidentes de faisabilité, les informations concernant la phase aiguë sont quasi inexistantes.

En phase tardive, sur des biopsies testiculaires chez un groupe de blessés médullaires par rapport à un groupe contrôle fertile, Hirsch a retrouvé un nombre plus élevé de cellules de Sertoli et une réduction du nombre de spermatides [12]. Chapelle a décrit également une atrophie testiculaire $[5,6]$.

Ces perturbations histologiques seraient expliquées par le choc spinal qui entraînerait une vasoconstriction, une nécrose de l'épithélium germinal puis une vasodilatation.

\section{Modifications des paramètres du spermogramme}

Les caractéristiques spermatiques ont été essentiellement étudiées chez le blessé médullaire chronique et sur des échantillons obtenus par vibromassage ou électroéjaculation. II est unanimement admis qu'une lésion médullaire a des répercussions négatives sur le spermogramme quel que soit le mode de recueil de sperme.

\section{a) Volume}

Pour la plupart des auteurs, il serait diminué du fait d'un dysfonctionnement des vésicules séminales. Toutefois le mode de recueil influence la quantité de sperme éjaculé. En effet, les volumes obtenus par masturbation ou par stimulation électrique sont inférieurs à ceux recueillis par vibromassage [15].

\section{b) Numération}

Dans la littérature, les résultats des études sont assez discordants, retrouvant soit une augmentation soit une réduction du nombre des spermatozoïdes [21], bien que la tendance générale soit à l'augmentation chez le blessé médullaire.

Le mode d'obtention du sperme a aussi son importance : le vibromassage permettrait d'obtenir un nombre plus important de spermatozoïdes [19].

\section{c) Mobilité}

Les études sur ce paramètre sont concordantes et montrent une baisse de la mobilité, essentiellement fléchante $[21,3,10]$ qui relèverait de différents mécanismes. L'hyperthermie scrotale [18], la stase importante dans les voies génitales, la présence d'anticorps anti-spermatozoïdes (ACAS) et celles de radicaux libres en sont les principaux $[2,3,10,21]$.

Encore une fois, il existe une influence du mode de recueil du sperme sur ce paramètre en faveur du vibromassage.

De plus, afin d'obtenir de meilleurs résultats en Assistance Médicale à la Procréation.(AMP), certains auteurs ont préconisé de « techniquer " le sperme dès son recueil.

\section{d) Tératospermie}

Des formes enroulées et la présence de restes cytoplasmiques en quantité plus importante ont été rapportées. Ces anomalies seraient liées à des infections récurrentes du tractus génital ainsi qu'à une altération de la spermiogenèse.

\section{e) Nécrospermie}

Toutes les études concordent : le sperme des patients blessés médullaires présente une nécrospermie expliquée par la présence d'ACAS [4, 16], par l'augmentation du temps de transit dans les voies génitales masculines, et par la présence de radicaux libres. Ces radicaux libres seraient essentiellement le produit de dégradation des polynucléaires neutrophiles [20].

D'autres facteurs peuvent également perturber la qualité spermatique :

- L'augmentation du délai entre la lésion et le recueil altère la qualité du sperme. Ainsi, certaines équipes préconisent de réaliser une congélation spermatique dès que possible après le traumatisme médullaire (dès le quinzième jour pour certains) [8].

- Le niveau lésionnel influe sur la mobilité avec un taux de spermatozoïdes mobiles plus important dans les lésions supérieures à T6 [17]. De même, le caractère incomplet de la lésion médullaire donnera un sperme de meilleure qualité qu'en cas de lésion complète.

- La qualité spermatique dépend aussi du mode et de la qualité de la vidange vésicale, le sondage intermittent étant plus favorable que les mictions par percussion et que la sonde vésicale à demeure.

- La toxicité de l'urine sur les gamètes en cas d'éjaculation rétrograde ou d'incontinence urinaire.

- L'effet iatrogène de certains médicaments (alpha bloquants, anti-inflammatoires non stéroïdiens, antibiotiques...).

\section{PRISE EN CHARGE DU COUPLE EN AMP}

En première intention, un bilan andrologique standard à la recherche d'antécédents urogénitaux antérieurs au traumatisme est à réaliser, ainsi qu'un bilan gynécologique permettant d'éliminer un ou plusieurs facteurs féminins d'infertilité.

Plusieurs situations sont possibles. Elles dépendent de l'existence d'une éventuelle éjaculation lors des rapports sexuels. Actuellement les rapports sexuels sont le plus souvent possibles, soit spontanés soit avec une aide médicamenteuse (inhibiteur de la phosphodiestérase ou injection intra caverneuse).

- Si une éjaculation est possible et que le sperme présente des caractéristiques normales, une conception naturelle est préconisée. Les résultats obtenus en pourcentage de grossesses sont identiques à ceux obtenus dans la population générale [7].

- Si une assistance médicalisée (vibromassage) est nécessaire pour obtenir une éjaculation et que les caractéristiques spermatiques sont normales, le couple a la possibilité de réaliser lui-même des inséminations à domicile. Les résultats concernant cette pratique sont évidemment difficiles à évaluer.

En revanche, dès qu'il existe des perturbations spermatiques, une prise en charge en AMP est le plus souvent nécessaire, le 
type de technique étant fonction du nombre de spermatozoïdes mobiles obtenus après préparation :

- Si le nombre de spermatozoïdes mobiles inséminables est supérieur à 1 million, les inséminations intra-utérines peuvent être envisagées. Les taux de grossesses par cycle (10 à $12,5 \%$ ) sont équivalents à ceux obtenus dans la population générale [22]. La technique de préparation du sperme est importante, le swim-up et le gradient de densité sont à privilégier.

- Si le nombre de spermatozoïdes inséminables est inférieur à 1 million, une prise en charge en FIV classique ou en FIVICSI est possible. En FIV classique, le taux de grossesse se situe entre 20 et $40 \%$ (taux de grossesses par cycle). Peu d'auteurs rapportent les résultats en FIV avec ICSI [7, 9, 14, 25], mais ceux-ci sont comparables à ceux obtenus dans la population générale.

- En cas d'échec d'éjaculation résistant au traitement, le recours à un prélèvement testiculaire chirurgical est indispensable pour réaliser une autoconservation spermatique. Dans un deuxième temps une prise en charge en AMP pourra être retenue, nécessitant une FIV avec ICSI du fait du faible nombre de gamètes.

Dans la plupart des cas, l'autoconservation du sperme est primordiale dès que sa réalisation est possible. Elle permet de conserver du sperme avant la survenue d'altérations spermatiques au cours du temps et liées principalement aux infections récurrentes et à la stase spermatique. Elle est particulièrement utile en cas :

- de difficultés de recueil,

- d'AMP avec injection intra cytoplasmique de spermatozoïde qui exige d'avoir des gamètes le jour de la fécondation,

- d'indication d'un stimulateur des racines sacrées qui modifie la réflectivité, conduit à des recueils de sperme plus difficiles, même médicalisés (vibromassage, EEJ), et nécessite parfois la réalisation d'un prélèvement chirurgical.

\section{CONCLUSIONS}

Une sensibilisation et une information des patients blessés médullaires à une prise en charge adaptée doivent être entreprises le plus rapidement possible.

II faut rester prudent en ce qui concerne le pronostic de procréation, les différents paramètres pouvant varier d'un couple à l'autre.

Lorsqu'une prise en charge médicale s'avère nécessaire, il faut essayer, quand les conditions le permettent, d'obtenir une éjaculation dans les conditions les plus simples possibles (vibromassage). En cas d'échec, l'escalade thérapeutique sera la plus progressive possible et ce n'est qu'en dernière extrémité que l'on devra recourir au prélèvement testiculaire.

La congélation spermatique devra être envisagée systématiquement de façon préventive, et sera faite le plus précocement possible étant données les dégradations du sperme (conséquences des infections génitales) et la fréquence des azoospermies spermatiques sécrétoires après traumatisme médullaire.

En cas d'échec de fécondation intra couple lié au seul problè- me masculin, nous pouvons informer le couple des possibilités de recours à un don de sperme.

Afin de réaliser cette approche et pour le plus grand bénéfice du patient, une collaboration efficace entre les différentes disciplines concernées (réanimateur, rééducateur fonctionnel, urologue et biologiste de l'AMP) est essentielle.

\section{REFERENCES}

1. AYERS J.W., MOINIPANH R., BENNETT C.J. et al. : Successful combination therapy with electroejaculation and in vitro fertilizationembryo transfer in the treatment of a paraplegic male with severe oligoasthenospermia. Fert. Steril., 1988, 49 : 1089-1090.

2. BERETTA G., CHELO E., MARZOTTO M. et al. : Anti-sperm antibodies in dysspermia in spinal cord injury patients. Arch. Ital. Urol. Androl., 1993, 65 : 189-192.

3. BRACKETT N.L., SANTA-CRUZ C. et al. : Sperm from spinal cord injury men lose motility faster than sperm from normal men : the effect is exacerbated at body compared to room temperature. J. Urol.,1997, $157:$ 2150-2153.

4. BRACKETT N.L., BLOCH W.E., LYNNE C.M. : Predictors of necrospermia in men with spinal cord injury. J. Urol., 1998, 159 : 844-847.

5. CHAPELLE P.A., ROBY-BRAMI A., YAKOVLEFF A. : Neurological correlations of ejaculation and testicular size in men with a complete spinal cord section. J. Neurol. Neurosurg. Psychiatry, 1988, $51: 197-202$.

6. CHAPELLE P.A., ROBY-BRAMI A., JONDET M. et al. : Trophic effects on testes in paraplegics. Paraplegia, 1993, $31: 576-583$.

7. DAHLBERG A., RUUTU M., HOVATTA O. : Pregnancy results from a vibrator application, electroejaculation, and a vas aspiration programme in spinal-cord injured men. Hum. Reprod.,1995, 10 : 20305-2307.

8. DAS S., SONI B.M., SHARMA S.D., GAZVANI R. : A case rapid deterioration in sperm quality following spinal cord injury. Spinal Cord, 2006, 44 : 56-58.

9. DE FORGE D., BLACKNER J., GARRITY C. et al. : Fertility following spinal cord injury : a systematic review. Spinal Cord, 2005 , $43: 693-703$.

10. DENYS P., SMAIL B.D., EVEN-SCHNEIDER A. : Les troubles génitosexuels du blessé médullaire. J. Soc. Biol., 2004, $198: 243$ 245.

11. FRANCOIS N. : Troubles génitaux-sexuels et procréation des blessés médullaires. Rev. Prat. (Paris), 1995, 45 : 2017-2021.

12. HIRSCH I.H., McCUE P., ALLEN J. : Quantitative testicular biopsy in spinal cord injured men comparison to fertile controls. $\mathrm{J}$. Urol.,1991, $146:$ 337-341.

13. HORNE M.W., PAUL D.P., MUNRO D. : Fertility studies in the human male with traumatic injuries of the spinal cord and cauda equina. New Engl. J. Med., 1948, $237:$ 959-961.

14. HUTLING C., ROSENLUND B., LEVI R. : Assisted ejaculation and in vitro fertilization in the treatment of infertile spinal cord-injured men : the role of intracytoplasmic sperm injection. Hum. Reprod., 1997, $12:$ 499-502.

15. JOUANNET P., FRANCOIS N., MAURY M. : Evaluation de la fertilité des hommes paraplégiques. J. Urol., 1983, $89: 169-171$.

16. MALLIDIS C., LIM T.C., HILL S.T. et al. : Necrospermia and chronic spinal cord injury. Fertil. Steril, 2000, $74: 221-227$.

17. MESNARD L. : La procréation des blessés médullaires. Intérêt de la décongélation préventive de sperme. Etude rétrospective à partir de 39 patients. Thèse pour le diplôme d'état de Docteur en médecine. Nantes, 2004.

18. MIEUSSET R., BUJAN L., MONDINAT C. et al. : Association of 
scrotal hyperthermia with impaired spermatogenesis in infertile men. Fertil. Steril., 1987, 48 : 1006-1011.

19. OHL D.A., SONKSEN J., MENGE A.C. et al. : Electroejaculation versus vibratory stimulation in spinal cord injured men : sperm quality and patient preference. J. Urol., 1997, $157: 2147-2149$.

20. PADRON O., BRACKETT N., SHARMA R.K. : Seminal reactive oxygen species and sperm motility and morphology in men with spinal cord injury. Fertil. Steril., 1997, $67: 1115-1120$.

21. PERKASH I., MARTIN D.E., WARNER H. et al. : Reproductive biology of paraplegics : results of semen collection, testicular biopsy and serum hormone evaluation. J. Urol., 1985, $134: 284-288$.

22. PRYOR J.L., KUNECK P.H., SELINA M. et al. : Delayed timing of intrauterine insemination results in a significantly improved pregnancy rate in female partners of quadriplegic men. Fertil. Steril., 2001, $76:$ 1130-1135.

23. SOBRERO A.J., HARLAN E.S., BLAIR J.B. : Technique for the induction of ejaculation in humans. Fertil. Steril., 1965, $16: 765-$ 767.

24. THOMAS R.J.S., MC LEISH G., MC DONALD I.A. et al. : Electroejaculation of the paraplegic man followed by pregnancy. Med. J. Aust., 1975, 2 : 798-799.

25. YAMAMOTO M., MOMOSE H., YAMADA K. : Fathering of a child with the assistance of electroejaculation in conjunction with intracytoplasmic sperm injection : case report. Spinal Cord, 1997, 35 : $179-180$

Manuscrit reçu : septembre 2007 ; accepté novembre 2007.

Communication FFER, octobre 2007, Amiens.

\section{ABSTRACT}

Biological strategies in assisted reproductive technologiesin spinal cord injury patients

\section{Manuel DEMAILLY, Henri COPIN, Philippe MERVIEL, Jacques PETIT, Isabelle HENRY}

Most men with spinal cord injury (SCI) men have fertility problems caused by anejaculation and decreased fertility of the ejaculate. There are two main causes for the impaired reproductive potential in $\mathrm{SCl}$ men: ejaculatory dysfunction and poor quality semen. However, current treatment techniques allow a large number of SCI males to achieve ejaculation (rectal electro-stimulation, penile vibrator stimulation).

Firstly, masturbation and/or penile vibrator are used at home allowing the couple to perform insemination themselves.

The semen of men with spinal cord injuries has commonly been characterised by small volume, abnormal count (low or high), decreased sperm mobility, increased formation of reactive oxygen species, sperm autoimmunity, necrospermia. This impairment is thought to be due to insufficient drainage, genitourinary infections and raised scrotal temperature. Testicular biopsy reveals varying degrees of tubule degeneration and decreased spermatogenetic activity.

Semen could be used for various assisted reproductive technologies such as intrauterine insemination, in vitro fertilisation (IVF) and microinsemination (ICSI).

The literature reports pregnancy rates by intrauterine insemination of about 15 to $20 \%$ per couple. Clinical pregnancy rates after IVF and ICSI techniques are $30 \%$ per cycle and these results are comparable to the clinical pregnancy rates when these techniques are performed for female infertility.

However, semen must be frozen as soon as possible after the injury and the patient must be informed about the various available assisted reproductive technologies.

Key Words : spinal cord injury, ejaculation, fertility, semen quality, ART 